\title{
Testes de vigor em sementes de Guazuma ulmifolia Lam.
}

\section{Tests of vigour in seeds of Guazuma ulmifolia Lam.}

\author{
Edilma Pereira Gonçalves ${ }^{1 *}$; Rinaldo César de Paula ${ }^{2}$; Maria Esmeralda S. P. Desmatlể
}

\section{Resumo}

O trabalho teve como objetivo estudar a eficiência dos testes de vigor para identificação do potencial fisiológico em sementes de Guazuma ulmifolia Lam. O estudo foi desenvolvido no Laboratório de Análise de Sementes - UNESP/Jaboticabal/SP, utilizando-se quatro lotes de sementes: sendo lotes I e II, coletados no município de Jaboticabal em 2000 e 2001, respectivamente lote III em Santa Rita do Passo Quatro e lote IV, em Luiz Antonio, ambos em 2001. Para superação da dormência, realizou-se a escarificação das sementes com ácido sulfúrico durante 50 minutos e lavagem em água corrente durante 20 minutos. Foram avaliados: teor de água, percentual de germinação, primeira contagem, índice de velocidade de germinação e condutividade elétrica, realizada com diferentes quantidades de sementes $(50,75,100)$, postas para embeber em copos de plástico contendo 50 e $75 \mathrm{~mL}$ de água deionizada, durante 72 horas à $25^{\circ} \mathrm{C}$. As sementes do lote III apresentaram potencial fisiológico superior às sementes dos demais lotes estudados. Para o teste de condutividade elétrica são necessários outros estudos, visando à possibilidade de uso como teste padrão de vigor para a espécie, enquanto que os testes de primeira contagem e índice de velocidade de germinação mostraram-se adequados para avaliação do vigor dos lotes.

Palavras-chave: Potencial fisiológico, condutividade elétrica, sementes florestais

\begin{abstract}
The work had as objective to verify the efficiency of vigour tests for identification of the physiologic potential in four lots of seeds of Guazuma ulmifolia Lam. The study was developed at the Laboratory of Seed Analysis - UNESP, Jaboticabal, SP. Four lots of seeds from three places of São Paulo state were used: lots I and II, collected in the municipal district of Jaboticabal in 2000 and 2001; lot II,: collected in Santa Rita do Passa Quatro and lot IV, Luiz Antonio, both in 2001. To break seed dormancy the seeds used in all the tests were escarified with sulfuric acid during 50 minutes, and washed in running water during 20 minutes. The evoluations were: seed moisture content, germination percentage, first count test, germination speed index and electric conductivity with different numbers of seeds $(50,75,100)$ put to soak in plastic containing 50 and $75 \mathrm{~mL}$ of water, to the temperature at $25^{\circ} \mathrm{C}$, during for 72 hours. The seeds of the lot originating from lot III, presented physiologic potential superior to the other studied lots. The tests of electric conductivity, first count, and index of germination speed were appropriate for evaluation of the physiologic potential of the lots. For the electric conductivity test other studies are necessary, involving a larger number of lots, seeking the use possibility a pattern vigour test, while the tests of first count and index of germination speed were appropriate for evaluation of the vigour.
\end{abstract}

Key words: Physiologic potential, electric conductivity, forest seeds

\footnotetext{
1 Eng. Agr. Dra em Produção e Tecnologia de Sementes, Bolsista CNPq/DCR. Universidade Estadual de Santa Cruz -UESC/Ihéus/ Bahia.Email: edilmapg@hotmail.com;

2 Prof. Dr. Adjunto da Universidade Estadual de São Paulo (UNESP)- Jaboticabal. Produção Vegetal.

3 Profa. Dra. Adjunto da Universidade Estadual de São Paulo UNESP/Jaboticabal.

* Autor para correspondência
} 


\section{Introdução}

A espécie Guazuma ulmifolia (Sterculiaceae) também conhecida vulgarmente como mutamba é uma árvore secundária inicial que atinge de 8 a 16 metros de altura, com tronco de 30 a $50 \mathrm{~cm}$ de diâmetro (TRAPE; OLIVEIRA, 1995). É uma espécie que forma associação micorrízica arbuscular e obtém benefício dessa associação para o seu crescimento, sendo, atualmente, bastante utilizada na recuperação de áreas degradadas (NISIZAKI; ZANGARO FILHO, 1996). A madeira é boa de trabalhar, macia, porém com pouco brilho, pouco resistente, mas utilizada para carroceria, marcenaria e caixotaria (PAULA; ALVES, 1997).

Nos laboratórios, rotineiramente, são feitos testes de germinação para a avaliação do potencial fisiológico das sementes. A utilização de sementes de boa qualidade constitui fator determinante para 0 êxito do empreendimento florestal, e o principal atributo da qualidade a ser considerado é a capacidade germinativa das sementes, pois, sem ela, a semente não tem valor para a semeadura, e dela também dependem a qualidade das mudas e o sucesso de um reflorestamento.

O teste de germinação apresenta menor sensibilidade para a diferenciação da qualidade entre lotes e, também, baixa correlação com a emergência das plântulas em campo. Por isso, têm-se intensificado estudos sobre testes de vigor, e os resultados têm sido satisfatórios no que diz respeito à identificação de possíveis diferenças na qualidade fisiológica de lotes que apresentam poder germinativo semelhante (PAIVA AGUERO, 1995). Também se buscam respostas complementares às fornecidas pelo teste de germinação e a possibilidade da obtenção de informações consistentes, de preferência em prazo de tempo relativamente curto.

Os testes de vigor fornecem índices mais sensíveis do potencial fisiológico, quando comparados ao teste de germinação. Desta forma, qualquer evento que preceda a perda do poder germinativo pode servir como base para o desenvolvimento de testes de vigor
(ASSOCIATION OF OFFICIAL SEED ANALYSTS, 1983).

A aplicação dos testes de vigor em sementes de espécies florestais é mais uma prática que permite estimar e comparar lotes de sementes para diferentes objetivos: avaliar o vigor de sementes entre matrizes, progênies e procedências; oferecer aos pesquisadores dados adicionais em uma fase inicial de um programa de melhoramento ou conservação genética (PIÑARODRIGUES; VALENTINI, 1995).

Programas internos de controle de qualidade desenvolvidos pelas entidades produtoras de sementes têm buscado o uso de testes que apresentem rapidez na obtenção dos resultados, e o teste de condutividade elétrica tem apresentando vantagens como rapidez e praticidade, mostrando-se promissor para avaliação do vigor em inúmeras espécies. Entretanto, grande parte destes testes destinados à avaliação da qualidade fisiológica de sementes são direcionados às sementes agrícolas, com menor ênfase para as espécies olerícolas, forrageiras e florestais.

No teste de condutividade elétrica, o período de embebição das sementes tem efeito marcante, principalmente por distinguir diferenças do potencial fisiológico entre lotes. Neste sentido, torna-se importante a condução de estudos que visem à redução do período de duração do teste, uma vez que a rapidez na obtenção das informações tem sido preconizada como fator fundamental para a dinamização dos programas de controle de qualidade interna implantados pelas empresas de sementes (DIAS, 1994).

Em sementes de Leucaena leucocephala, diferentes tempos de embebição foram estudados e o período de 72 horas apresentou tendência de maior efetividade na separação dos lotes, embora, em todos os tempos testados (24, 48 e $72 \mathrm{~h}$ ), tivessem sido detectadas diferenças significativas entre lotes (DUBOC et al., 1993).

Segundo Amaral, Villela e Peske (1997) o período de 24 horas é suficiente para estimar a qualidade 
fisiológica em sementes de Cordia trichotoma, quando se utilizam quatro repetições de 50 sementes em 75mL de água deionizada, permitindo a separação dos lotes em diferentes níveis de vigor.

Barbedo e Cícero (1998) verificaram que o teste de condutividade elétrica durante 24 horas de embebição a $25^{\circ} \mathrm{C}$ favoreceu uma estimativa do potencial germinativo de lotes de sementes de Inga uruguensis. Zucareli (1999) afirmou que o teste de condutividade elétrica realizado com 10 sementes em $75 \mathrm{~mL}$ de água destilada por um período de 4 horas mostrou-se eficiente na separação de lotes de sementes de farinha-seca (Albizia hasslerii (Chodat). Burr).

O desempenho de sementes de duas populações de aroeira (Myracrodruon urundeuva) foi estudado por Moraes, Oliveira e Freitas (1997), por meio do teste de condutividade elétrica, utilizando-se 50 sementes e $75 \mathrm{~mL}$ de água a $25^{\circ} \mathrm{C}$ durante 24 horas. Os autores verificaram que valores acima de $720 \mathrm{a} \mu \mathrm{S} /$ $\mathrm{cm} / \mathrm{g}$ da condutividade elétrica corresponderam a valores nulos de germinação, que indicam sementes totalmente deterioradas.

O teste de condutividade elétrica se mostrou eficiente na diferenciação de lotes de sementes de Dalbergia nigra, podendo ser conduzido com amostras de 50 sementes, embebidas por pelo menos 36 horas em $75 \mathrm{ml}$ de água deionizada, a $25^{\circ} \mathrm{C}$ (MARQUES, 2001).

Baseado no que foi exposto, o trabalho teve como objetivo verificar a eficiência dos testes de vigor para identificação do potencial fisiológico em quatro lotes de sementes de mutamba (Guazuma ulmifolia Lam.).

\section{Material e Métodos}

O trabalho foi desenvolvido no Laboratório de Análise de Sementes da Faculdade de Ciências Agrárias e Veterinárias - UNESP, Campus de Jaboticabal, SP.

As sementes de mutamba (Guazuma ulmifolia Lam.) foram provenientes de três locais do estado de São Paulo: Lotes I e II, coletados no município de Jaboticabal em 2000 e 2001; Lote III, coletado em Santa Rita Passa Quatro e Lote IV, coletado em Luiz Antonio, ambos em 2001.

Os frutos provenientes de Jaboticabal e Luiz Antonio foram colhidos pela agitação dos ramos de 10 árvores. Em seguida, foram levados para o laboratório, onde as sementes foram extraídas dos frutos a partir das fendas localizadas na sua porção inferior, com o auxílio de uma espátula fina, para evitar danos mecânicos. As sementes provenientes de Santa Rita do Passa Quatro (2001) foram colhidas de 12 árvores na respectiva localidade.

Com a finalidade de se obterem lotes com maior homogeneização, antes da instalação dos testes, as sementes foram classificadas por peneira com diâmetro $\geq 2,0 \mathrm{~mm}$ e armazenadas em câmara fria à $10^{\circ} \mathrm{C}$ até o início dos testes. Em todos os testes foram utilizadas sementes escarificadas com ácido sulfúrico durante 50 minutos, conforme recomendação de Araújo Neto (1997). Em seguida, foram lavadas em água corrente durante 20 minutos.

\section{Determinação do teor de água}

Foi efetuado pelo método estufa a $105^{\circ} \mathrm{C} \pm 3^{\circ} \mathrm{C}$, durante 24 horas, conforme (BRASIL, 1992), utilizando-se duas amostras de 0,30g de sementes para cada lote, com aproximadamente 50 sementes. Os resultados foram expressos em porcentagem média para cada lote.

\section{Teste de germinação}

Foi realizado com 200 sementes (quatro repetições de 50 sementes) para cada lote, distribuídas em caixas plásticas tipo "gerbox", contendo como substrato papel de filtro umedecido com quantidade de água 2,5 vezes o peso do papel, e colocadas para germinar em temperatura de $30^{\circ} \mathrm{C}$, com fotoperíodo de 8 horas. As contagens foram realizadas a partir do $5^{0}$ dia após a semeadura até 28 dias após a 
instalação do teste, segundo os critérios de Araújo Neto (1997). Foram computadas as porcentagens de plântulas normais para cada repetição, obtendo-se os dados médios por lote.

\section{Teste de primeira contagem de germinação}

Conduzido conjuntamente com o teste de germinação, consistindo na contagem de plântulas normais encontradas na primeira contagem do referido teste, que foi realizada no $5^{0}$ dia após semeadura, considerando-se as plântulas normais, segundo recomendações de (ARAÚJO NETO, 1997). Os resultados foram expressos em porcentagem média de plântulas normais por lote.

\section{Índice de velocidade de germinação}

Realizado juntamente com o teste padrão de germinação. As avaliações das plântulas normais foram realizadas diariamente, à mesma hora, a partir da primeira contagem da germinação até o último dia de contagem de germinação (MAGUIRE, 1962).

\section{Condutividade elétrica}

Para cada lote, foram pesadas quatro repetições com diferentes números de sementes $(50,75,100)$. Em seguida, as sementes foram postas para embeber em copos de plástico $(200 \mathrm{~mL})$ contendo 50 e $75 \mathrm{~mL}$ de água deionizada $(2,5 \mu \mathrm{mhos} / \mathrm{cm}$ de condutividade) e mantidas em temperatura de $25^{\circ} \mathrm{C}$ durante 72 horas. A condutividade elétrica da solução foi avaliada após 30 min, 2h, 4h, 6h, 8h, 16h, 24h, 32h, 48h e 72h de embebição, totalizando 10 períodos de avaliação. Após cada período de embebição, a condutividade elétrica da solução foi avaliada por meio de leituras em condutivímetro DIGIMED DM - 31. O resultado da leitura foi dividido pela massa seca(g) da amostra e os valores, expressos em $\mu \mathrm{mhos} / \mathrm{cm} / \mathrm{g}$ de sementes.

\section{Delineamento estatístico}

Os resultados de germinação, primeira contagem, índice de velocidade de germinação, foram avaliados seguindo delineamento inteiramente casualizado com quatro tratamentos (lotes) e quatro repetições, e as médias foram comparadas pelo teste Tukey, a 5\% de probabilidade.

Para a condutividade elétrica, o delineamento experimental foi inteiramente casualizado, em parcelas subdivididas para os períodos de embebição. Os resultados foram analisados por esquema fatorial (4 $\mathrm{x} 3 \times 2$ ), sendo quatro lotes $\mathrm{x}$ três quantidades de sementes $\mathrm{x}$ dois volumes de água, e as parcelas foram representadas por 10 períodos de embebição das sementes.

Os graus de liberdade para a interação entre lote, quantidade de sementes, volume de água e tempo de embebição foram decompostos pelo uso da análise de regressão polinomial até terceiro grau. O modelo escolhido correspondeu aquele de maior grau que, na decomposição dos graus de liberdade, apresentou significância estatística a 5\% pelo teste $\mathrm{F}$.

As médias de condutividade elétrica entre os lotes para cada combinação, número de sementes, volume de água e tempo de embebição foram comparadas pelo teste de Tukey, a 5\% de probabilidade.

\section{Resultados e Discussão}

$\mathrm{Na}$ Tabela 1, encontra-se a avaliação inicial da qualidade fisiológica dos lotes de sementes de mutamba. Com relação aos dados referentes ao teor de água das sementes nos diferentes lotes, verificase que apresentaram pequenas variações $(9,2$ a $10,0 \%$ ). Este fato é importante para a execução dos testes, considerando-se que a uniformização do teor de água das sementes é imprescindível para a padronização das avaliações e obtenção de resultados consistentes (KRZYZANOWSKI; FRANÇA NETO, 1991; HAMPTON; TEKRONY, 1995; MARCOS FILHO, 1999). 
Tabela 1. Teor de água (\%) e resultados da qualidade fisiológica inicial dos lotes de sementes de mutamba (Guazuma ulmifolia) avaliada pelos testes de primeira contagem (\%), germinação (\%) e índice de velocidade de germinação (IVG).

\begin{tabular}{ccccc}
\hline & Teor de água (\%) & $\begin{array}{c}\text { Primeira } \\
\text { Contagem (\%) }\end{array}$ & Germinação (\%) \\
\hline Lote I & 10,0 & $33,0 \mathrm{~b}$ & $63,0 \mathrm{a}$ & $5,10 \mathrm{ab}$ \\
Lote II & 9,5 & $36,0 \mathrm{~b}$ & $58,0 \mathrm{a}$ & $4,68 \mathrm{~b}$ \\
Lote III & 9,2 & $58,0 \mathrm{a}$ & $73,0 \mathrm{a}$ & $6,75 \mathrm{a}$ \\
Lote IV & 9,3 & $43,5 \mathrm{ab}$ & $64,5 \mathrm{a}$ & $5,41 \mathrm{ab}$ \\
Média & & & 5,48 \\
CV & & 42,63 & 64,63 & 16,94 \\
\hline
\end{tabular}

Médias seguidas de mesma letra na linha não diferem entre si, pelo teste de Tukey, em nível de $5 \%$ de probabilidade

O teor de água elevado pode favorecer o desempenho das sementes no processo de germinação, pois as sementes mais úmidas, dentro de certos limites, germinam mais rapidamente. Os teores de água das sementes dos lotes analisados não variaram muito, sendo possível compará-los entre si.

Verifica-se que os lotes não diferiram entre si pelo teste de germinação. Entretanto, o teste de primeira contagem indicou que os lotes I e II diferiram estatisticamente do lote III. Normalmente, a capacidade dos testes de laboratório para estimar o potencial de emergência das plântulas diminui à medida que as condições de ambiente vão se desviando das mais adequadas, tornando-se praticamente nula sob condições extremamente desfavoráveis.

Os testes de primeira contagem de germinação e o índice de velocidade de germinação se mostraram mais sensíveis para detectar diferenças entre os lotes que o teste de germinação. Segundo Nakagawa (1999), o teste de primeira contagem de germinação, muitas vezes, expressa melhor as diferenças de velocidade de germinação entre lotes do que os índices de velocidade de germinação, e ainda é um teste considerado menos trabalhoso que o de velocidade de germinação. De acordo com Valentini e Piña-
Rodrigues (1995), o teste de primeira contagem apresenta eficiência reduzida quanto à detecção de pequenas diferenças de vigor.

Para o índice de velocidade de germinação, foi observado que o lote II apresentou-se como o de menor potencial fisiológico, não diferindo estatisticamente dos lotes I e IV. Embora a redução da velocidade da germinação não esteja relacionada com os primeiros eventos do processo de deterioração de sementes, este teste tem sido bastante utilizado apresentando resultados coerentes com o potencial fisiológico das sementes.

Marcos Filho (1998) ressalta a importância de se fazer uso de mais de um teste para determinar o vigor de lotes de sementes. Isso se deve à influência dos métodos adotados e do uso de situações específicas de estresse para estimar o comportamento relativo dos lotes (TEKRONY; EGLI, 1977; CARVALHO; NAKAGAWA, 2000).

Os valores de $\mathrm{F}$ obtidos na análise de variância, para a condutividade elétrica de massa $(\mu \mathrm{mhos} / \mathrm{cm} /$ g) à temperatura de $25^{\circ} \mathrm{C}$, encontram-se na Tabela 2 , e permitem constatar que ocorreram efeitos significativos de todas as fontes de variação, excetuando, número de sementes e sua interação com volume de água. 
Tabela 2. Condutividade elétrica de massa ( $\mu \mathrm{mhos} / \mathrm{cm} / \mathrm{g}$ ) de quatro lotes de sementes de mutamba (Guazuma ulmifolia) em amostras de 50, 75 e 100 sementes embebidas em diferentes volumes de água e períodos de embebição.

\begin{tabular}{|c|c|c|c|c|c|c|c|c|c|}
\hline \multirow{2}{*}{\multicolumn{2}{|c|}{$\begin{array}{l}\text { Tempo de } \\
\text { embebição/ } \\
\text { Número de } \\
\text { sementes }\end{array}$}} & \multicolumn{8}{|c|}{ Volume de água } \\
\hline & & \multicolumn{4}{|c|}{$50 \mathrm{~mL}$} & \multicolumn{4}{|c|}{$75 \mathrm{~mL}$} \\
\hline & & Lote I & Lote II & Lote III & Lote IV & Lote I & Lote II & Lote III & Lote IV \\
\hline \multirow{3}{*}{$0,5 \mathrm{~h}$} & $50 \mathrm{sem}$ & $46,04 b$ & $91,79 \mathrm{ab}$ & $176,83 a$ & $75,34 b$ & $43,81 \mathrm{~b}$ & $39,90 \mathrm{~b}$ & $74,73 a$ & $52,74 \mathrm{ab}$ \\
\hline & $75 \mathrm{sem}$ & $69,24 \mathrm{ab}$ & $102,29 \mathrm{a}$ & $52,62 \mathrm{ab}$ & 76,78ab & $42,97 \mathrm{~b}$ & $56,68 \mathrm{ab}$ & $56,90 \mathrm{~b}$ & $76,66 a$ \\
\hline & 100 sem & $44,38 b$ & $117,37 \mathrm{a}$ & $149,93 \mathrm{a}$ & $113,81 \mathrm{a}$ & $36,57 \mathrm{~b}$ & $69,85 \mathrm{ab}$ & $66,68 \mathrm{ab}$ & $94,81 \mathrm{a}$ \\
\hline \multirow{3}{*}{$2 \mathrm{~h}$} & $50 \mathrm{sem}$ & $61,21 \mathrm{~b}$ & $126,35 \mathrm{ab}$ & $234,39 a$ & $91,96 a b$ & $54,94 \mathrm{~b}$ & $63,11 \mathrm{~b}$ & $114,61 \mathrm{a}$ & $68,48 \mathrm{~b}$ \\
\hline & $75 \mathrm{sem}$ & $88,75 b$ & $129,11 \mathrm{a}$ & $66,06 \mathrm{~b}$ & 97,39ab & $55,07 \mathrm{~b}$ & $73,58 \mathrm{ab}$ & $66,69 \mathrm{ab}$ & $90,83 \mathrm{a}$ \\
\hline & 100 sem & $61,75 b$ & $138,00 \mathrm{a}$ & $158,91 \mathrm{a}$ & $126,03 \mathrm{a}$ & $50,47 \mathrm{~b}$ & $82,26 a b$ & $70,49 \mathrm{ab}$ & $101,86 \mathrm{a}$ \\
\hline \multirow{3}{*}{$4 \mathrm{~h}$} & $50 \mathrm{sem}$ & $74,29 b$ & $130,07 \mathrm{ab}$ & $217,55 \mathrm{a}$ & $104,99 \mathrm{ab}$ & $62,81 \mathrm{a}$ & $74,89 \mathrm{a}$ & $116,65 \mathrm{a}$ & $77,13 a$ \\
\hline & $75 \mathrm{sem}$ & $101,00 \mathrm{bc}$ & $155,36 a$ & $70,98 \mathrm{c}$ & $116,77 \mathrm{ab}$ & $57,96 \mathrm{~b}$ & $80,26 a b$ & $68,13 b$ & $97,90 \mathrm{a}$ \\
\hline & 100 sem & $74,85 \mathrm{~b}$ & $150,42 \mathrm{a}$ & $158,71 \mathrm{a}$ & $142,00 \mathrm{a}$ & $56,55 \mathrm{~b}$ & $92,35 b$ & $73,34 b$ & $112,11 \mathrm{a}$ \\
\hline \multirow{3}{*}{$6 \mathrm{~h}$} & $50 \mathrm{sem}$ & $96,01 b$ & $139,58 \mathrm{ab}$ & 205,87 & $127,03 \mathrm{ab}$ & $77,73 \mathrm{a}$ & $92,96 a$ & $114,67 \mathrm{a}$ & $91,37 \mathrm{a}$ \\
\hline & $75 \mathrm{sem}$ & $117,12 \mathrm{~b}$ & $176,30 \mathrm{a}$ & $85,83 \mathrm{c}$ & $131,81 \mathrm{~b}$ & $73,43 b$ & $93,68 \mathrm{ab}$ & $71,97 \mathrm{~b}$ & $111,50 \mathrm{a}$ \\
\hline & 100 sem & $97,05 b$ & $167,38 \mathrm{a}$ & $152,53 \mathrm{a}$ & $166,15 \mathrm{a}$ & $75,76 b$ & $104,18 b$ & $77,81 b$ & $127,25 \mathrm{a}$ \\
\hline \multirow{3}{*}{$8 \mathrm{~h}$} & $50 \mathrm{sem}$ & $128,19 \mathrm{a}$ & $146,50 \mathrm{a}$ & $197,98 \mathrm{a}$ & $165,64 a$ & $100,90 \mathrm{a}$ & $100,54 \mathrm{a}$ & $113,97 \mathrm{a}$ & $116,36 \mathrm{a}$ \\
\hline & $75 \mathrm{sem}$ & $120,58 b$ & $179,67 \mathrm{a}$ & $91,44 \mathrm{c}$ & $163,99 a$ & $75,81 \mathrm{~b}$ & $97,86 b$ & $74,35 b$ & $136,62 \mathrm{a}$ \\
\hline & 100 sem & $130,03 \mathrm{c}$ & $173,93 \mathrm{ab}$ & $154,73 b c$ & $197,92 \mathrm{a}$ & $101,18 b$ & $108,41 b$ & $79,76 b$ & $149,48 \mathrm{a}$ \\
\hline \multirow{3}{*}{$16 \mathrm{~h}$} & $50 \mathrm{sem}$ & $163,80 \mathrm{a}$ & $164,10 \mathrm{a}$ & $203,40 \mathrm{a}$ & $202,16 a$ & $130,34 \mathrm{a}$ & $119,13 \mathrm{a}$ & $115,93 a$ & $142,67 \mathrm{a}$ \\
\hline & $75 \mathrm{sem}$ & $187,58 b$ & $246,73 a$ & $109,02 \mathrm{c}$ & $198,46 b$ & $120,78 b$ & $140,50 \mathrm{ab}$ & $84,49 c$ & $167,53 \mathrm{a}$ \\
\hline & 100 sem & $155,18 b$ & $244,95 \mathrm{a}$ & $163,24 b$ & $240,78 \mathrm{a}$ & $123,56 b c$ & $150,73 \mathrm{ab}$ & $88,88 \mathrm{c}$ & $177,01 \mathrm{a}$ \\
\hline \multirow{3}{*}{$24 \mathrm{~h}$} & $50 \mathrm{sem}$ & $170,37 \mathrm{a}$ & $186,89 \mathrm{a}$ & $220,80 \mathrm{a}$ & $211,31 \mathrm{a}$ & $134,61 \mathrm{a}$ & $142,07 \mathrm{a}$ & $130,56 a$ & $148,86 \mathrm{a}$ \\
\hline & $75 \mathrm{sem}$ & $187,06 \mathrm{~b}$ & $252,055 \mathrm{a}$ & $132,47 \mathrm{c}$ & $189,80 \mathrm{~b}$ & $121,72 b c$ & $142,02 \mathrm{ab}$ & $99,30 \mathrm{c}$ & $170,59 \mathrm{a}$ \\
\hline & 100 sem & $164,50 \mathrm{~b}$ & $234,05 \mathrm{a}$ & $178,59 \mathrm{~b}$ & $236,65 \mathrm{a}$ & $127,45 b c$ & $150,26 \mathrm{ab}$ & $103,54 \mathrm{c}$ & $174,08 \mathrm{a}$ \\
\hline \multirow{3}{*}{$32 \mathrm{~h}$} & $50 \mathrm{sem}$ & $182,50 \mathrm{a}$ & $212,12 \mathrm{a}$ & $240,98 \mathrm{a}$ & $214,76 \mathrm{a}$ & $142,20 \mathrm{a}$ & $173,68 \mathrm{a}$ & $153,01 \mathrm{a}$ & $153,18 \mathrm{a}$ \\
\hline & $75 \mathrm{sem}$ & $199,01 b c$ & $255,36 a$ & $166,23 \mathrm{c}$ & $206,14 b$ & $132,44 b$ & $139,77 b$ & $122,22 \mathrm{~b}$ & $175,21 \mathrm{a}$ \\
\hline & 100 sem & $173,98 \mathrm{~b}$ & $237,57 \mathrm{a}$ & $204,96 \mathrm{ab}$ & $244,48 \mathrm{a}$ & $136,44 b$ & $150,22 \mathrm{ab}$ & $124,64 b$ & $177,79 \mathrm{a}$ \\
\hline \multirow{3}{*}{$48 \mathrm{~h}$} & 50 sem & $184,57 \mathrm{a}$ & $214,46 \mathrm{a}$ & $239,62 \mathrm{a}$ & $215,55 \mathrm{a}$ & $141,82 \mathrm{a}$ & $174,91 \mathrm{a}$ & $156,77 \mathrm{a}$ & $155,60 \mathrm{a}$ \\
\hline & $75 \mathrm{sem}$ & $194,93 \mathrm{bc}$ & $256,31 \mathrm{a}$ & $171,45 \mathrm{c}$ & $213,07 b$ & $133,55 b$ & $144,06 b$ & $126,69 b$ & $175,21 \mathrm{a}$ \\
\hline & 100 sem & $149,08 \mathrm{~b}$ & $238,40 \mathrm{a}$ & $205,73 \mathrm{a}$ & $243,52 \mathrm{a}$ & $125,40 \mathrm{~b}$ & $154,18 \mathrm{ab}$ & $127,50 \mathrm{~b}$ & $177,60 \mathrm{a}$ \\
\hline \multirow{3}{*}{$72 \mathrm{~h}$} & $50 \mathrm{sem}$ & $182,84 b$ & $218,71 \mathrm{ab}$ & $246,68 \mathrm{a}$ & $216,03 \mathrm{ab}$ & $142,46 a$ & $175,60 \mathrm{a}$ & $161,73 a$ & $153,56 a$ \\
\hline & $75 \mathrm{sem}$ & $194,93 b c$ & $255,87 \mathrm{a}$ & $174,19 \mathrm{c}$ & $213,07 \mathrm{~b}$ & $133,35 b$ & $144,18 b$ & $130,91 b$ & $175,33 \mathrm{a}$ \\
\hline & 100 sem & $161,19 b$ & $238,12 \mathrm{a}$ & $210,73 a$ & $244,17 \mathrm{a}$ & $126,93 b$ & $154,33 \mathrm{ab}$ & $130,51 b$ & $177,52 \mathrm{a}$ \\
\hline
\end{tabular}

Valores seguidos de mesma letra, para cada combinação lote, tempo de embebição, número de sementes e volume de água, não diferem significativamente entre si em nível de $5 \%$ de probabilidade pelo teste de Tukey. 
Os teores de água das sementes antes da realização do teste foram: 10,2\% para o lote I, 9,7\% para o lote II, 9,8\% para o lote III e 9,6\% para o lote IV. Para o teste de condutividade elétrica em soja, a recomendação para o teor de água das sementes, antes da condução do teste, é de 11 a $17 \%$ (ASSOCIATION OF OFFICIAL SEED ANALYSTS, 1983), enquanto Tao (1978) e Loeffler; Tekrony e Egli (1988) recomendaram o uso de sementes com teor de água entre 13 e 18\%.

Embora os valores dos teores de água $(9,6$ a $10,2 \%)$ das amostras do experimento estejam um pouco abaixo do recomendado para sementes de soja, valores muitos altos de condutividade elétrica somente começam a ser encontrados quando o teor de água inicial é inferior a 8,8\%, de acordo com Tao (1978), Hampton (1992); Prete (1992) e Vanzolini e Nakagawa (1999). Além disso, os valores encontrados no trabalho não variaram muito entre as amostras analisadas, podendo ser comparados entre si. Marques (2001) utilizou sementes de jacarandá-da-bahia (Dalbergia nigra) com teores de água variando de $9,4 \%$ a $10,6 \%$, e considerou que estas diferenças não afetariam os resultados dos testes.

$\mathrm{Na}$ Tabela 2, encontram-se os dados da condutividade elétrica de massa $(\mu \mathrm{mhos} / \mathrm{cm} / \mathrm{g})$ à temperatura de $25^{\circ} \mathrm{C}$, com diferentes quantidades de sementes, volumes de água e períodos de embebição. De uma forma geral, os resultados obtidos, nas diversas combinações, indicaram um aumento progressivo dos lixiviados com o decorrer do período de embebição, o que se mostra coerente com as observações feitas por Loeffler, Tekrony e Egli (1988), Marcos Filho et al. (1990), Dias, Vieira e Bhéring (1996) e Marques (2001). Constatou-se também que, com o aumento do volume de água, quando se mantiveram constantes os outros fatores (quantidade de sementes e período de embebição), estabeleceu-se, em geral, relação direta com a diminuição do valor da lixiviação. Loeffler (1981) verificou maiores valores de condutividade elétrica em menores volumes de água, e atribuiu isso ao fato de que a embebição, em um volume maior de água, implica maior diluição dos lixiviados.
Na Figura 1A e na Tabela 2, estão os resultados obtidos com o teste de condutividade elétrica para a combinação 50 sementes e $50 \mathrm{~mL}$ de água. Observase que, nos primeiros períodos de embebição, os valores de condutividade apresentaram diferenças significativas entre os lotes estudados, e que estas diferenças desapareceram a partir do período de 8 horas de embebição. Resultados similares foram observados quando se utilizou o mesmo número de sementes com $75 \mathrm{~mL}$ de água. Entretanto, houve antecipação do período de embebição para 4 horas.

Conforme mostram as Figuras $1 \mathrm{~A}$ e $2 \mathrm{~A}$ (50 sementes, com 50 e $75 \mathrm{~mL}$ de água), não houve ordenação dos lotes quanto ao vigor, dificultando indicações mais precisas sobre o potencial fisiológico de cada lote, principalmente quando se aumentou o volume de água.

De uma forma geral, para as combinações com 50 sementes nos diferentes volumes de água estudados (50 e $75 \mathrm{~mL})$, não foram verificadas diferenças estatísticas no vigor dos lotes. Provavelmente, o tamanho das sementes tenha influenciado esses resultados, pois, quando aumentou o número de sementes, foram observados resultados contraditórios aos encontrados com estas combinações (Figura 1C).

Observando os dados obtidos para o teste de condutividade elétrica, na combinação 100 sementes em 50mL de água, o lote I aparece como o de melhor potencial fisiológico, já nos 30 minutos de embebição (Tabela 2 e Figura 1C). A partir de 8 horas de embebição, o lote III, apresentou-se com menor quantidade de lixiviados em relação aos lotes II e IV, sendo que em alguns períodos, não diferiu estatisticamente.

Analisando os resultados da combinação de 75 sementes e 50mL de água (Tabela 2 e Figura 1B), verificou-se que, a partir do período de 2 horas de embebição, foi possível uma separação dos lotes. $\mathrm{O}$ lote III apresentou menor quantidade de lixiviados, não diferindo estatisticamente em alguns períodos do lote I. A partir de 16 horas de embebição, o lote II apresentou quantidades de lixiviados superiores aos 
demais lotes, diferindo estatisticamente. Os resultados encontrados nesta combinação estão coerentes com os obtidos no índice de velocidade de germinação (Tabela 1). Apesar de o índice de velocidade de germinação não estar relacionado entre os primeiros eventos do processo de deterioração de sementes, este teste foi sensível para classificar os lotes estudados.
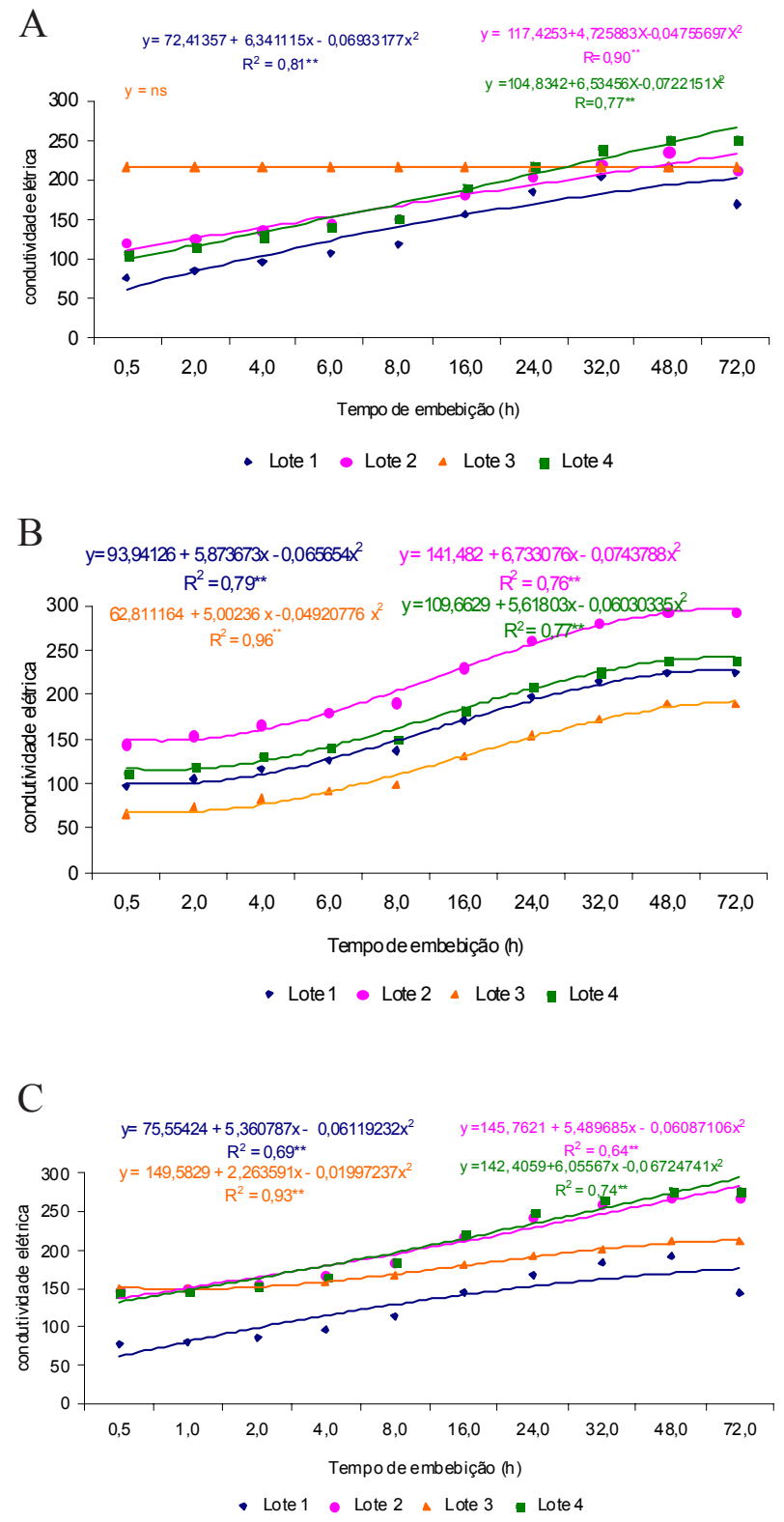

Figura 1. Condutividade elétrica de massa $(\mu \mathrm{mhos} / \mathrm{cm} / \mathrm{g})$ em sementes de mutamba (Guazuma ulmifolia) pertencentes a quatro lotes, embebidas à $25^{\circ} \mathrm{C}$. Amostras com (A) 50 sementes, (B) 75 sementes e (C) 100 sementes, embebidas em $50 \mathrm{~mL}$ de água, em função de diferentes períodos de embebição.
De acordo com Roberts (1973) e Anderson e Baker (1983), durante a deterioração da semente, ocorre a perda de integridade das membranas, nas organelas que compõem as células, resultando em exsudação de íons, açúcares e metabólitos durante o processo de embebição. Para todas as sementes, a integridade das membranas é incompleta durante, no mínimo, alguns minutos após a embebição; contudo, passado o tempo, essa situação se altera com a retomada natural da sua configuração estável, possivelmente por meio da restauração promovida por um mecanismo enzimático ainda não perfeitamente identificado (PIANA; TILLMANN; MINAMI, 1995).

Em sementes deterioradas, este mecanismo de reparo está ausente ou é ineficiente ou, ainda, as membranas acham-se tão profundamente danificadas que o reparo não se realiza (BEWLEY; BLACK, 1994). É o que provavelmente ocorreu com as sementes dos lotes II e IV. Por ser lotes de germinação inicial inferior aos demais, as membranas celulares estariam mais desorganizadas e, durante a embebição, liberariam maiores quantidades de lixiviados, pois estes lotes apresentaram sempre maiores valores de condutividade elétrica nos períodos estudados, quando utilizou 75 sementes e $50 \mathrm{~mL}$ de água.

Examinando a Tabela 2, para a combinação 75 sementes em $75 \mathrm{~mL}$ de água, observou-se que os lotes I e III apresentaram menor quantidade de lixiviados, a partir de 4 horas de embebição, entretanto, em alguns períodos não diferiram estatisticamente do lote II. Resultado similar foi encontrado utilizando $50 \mathrm{~mL}$ de água com este mesmo número de sementes. Quando se utilizaram 100 sementes e $75 \mathrm{~mL}$ de água, os lotes I e III, aparecem novamente com menores quantidades de lixiviados, a partir de 6 horas de embebição (Figura 2B). 
Nas Figuras 1C e 2C, encontram-se os resultados da condutividade elétrica para a combinação 100 sementes embebidas em $50 \mathrm{~mL}$ e $75 \mathrm{~mL}$ de água, respectivamente. Ocorreu uma mudança na estratificação dos lotes III e I, sendo que, com $75 \mathrm{~mL}$, a seqüência foi similar às encontradas em outras combinações.

De uma forma geral, fixando-se o número de sementes em 75 , com $50 \mathrm{~mL}$ ou $75 \mathrm{~mL}$, foi possível constatar que os períodos de embebição de seis horas e quatro horas mostraram concordância quanto à ordenação dos lotes e à possibilidade de redução do período de condicionamento das sementes de mutamba (Tabela 2). Alguns pesquisadores já afirmaram a possibilidade de redução do período de condicionamento para algumas hortaliças. Torres et al. (1998) recomendaram o período de quatro horas para as sementes de maxixe. Este mesmo período foi recomendado para sementes de repolho (LOOMIS; SMITH, 1980). Vanzolini e Nakagawa (1999) observaram que, com 3 horas, já foi possível a separação do lote de qualidade inferior de sementes de amendoim.

A duração do período em que ocorre a lixiviação varia consideravelmente entre espécies e cultivares e está, provavelmente, relacionada com o tamanho das sementes (HALMER; BEWLEY, 1984). No caso de sementes pequenas, a lixiviação máxima pode ocorrer num período inferior a duas horas (MURPHY; NOLAND, 1982), ao passo que em sementes maiores, como soja, verificou-se aumento de lixiviação de 24 a 30 horas após o início da embebição, a uma temperatura de $25^{\circ} \mathrm{C}$ (LOEFFLER et al., 1988).

Em sementes de Leucaena leucocephala, diferentes tempos de embebição foram estudados e o período de 72 horas apresentou tendência de maior efetividade na separação dos lotes (DUBOC et al., 1993). Por sua vez, Amaral, Villela e Peske (1997) verificaram que o período de 24 horas é suficiente para estimar o potencial fisiológico em sementes de Cordia trichotoma.

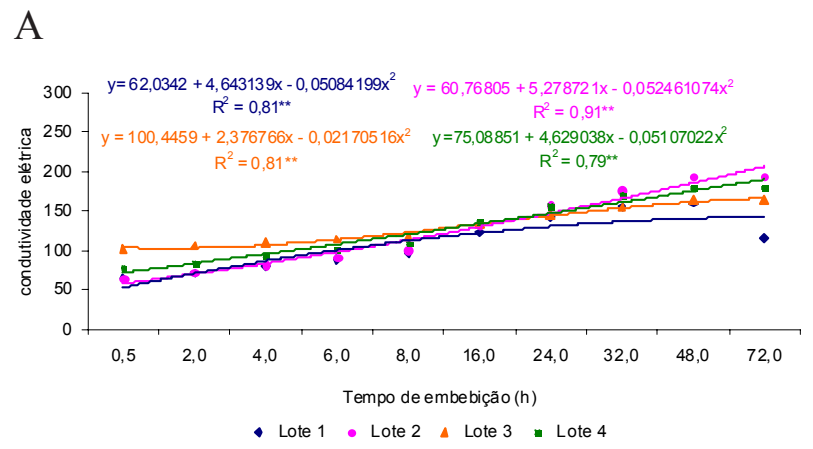

$B$

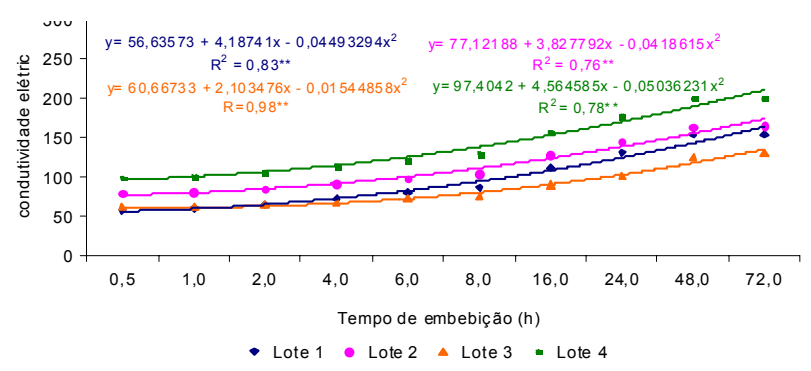

C

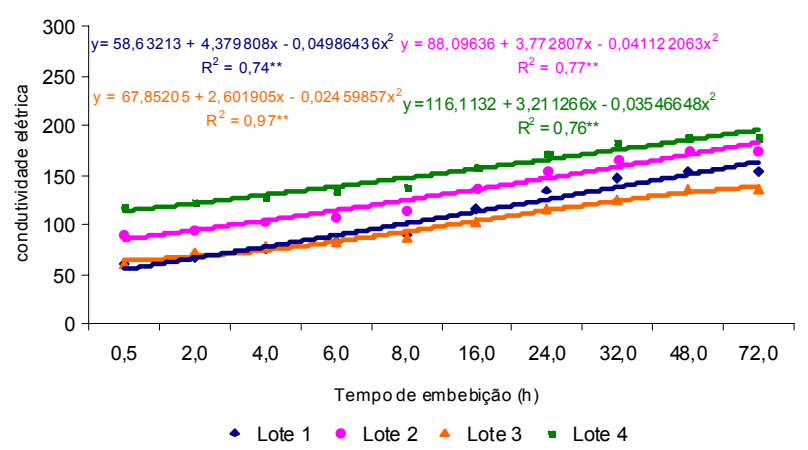

Figura 2. Condutividade elétrica de massa $(\mu \mathrm{mhos} / \mathrm{cm} / \mathrm{g})$ em sementes de mutamba (Guazuma ulmifolia) pertencentes a quatro lotes, embebidas à $25^{\circ} \mathrm{C}$. Amostras com (A) 50 sementes, (B) 75 sementes e (C) 100 sementes, embebidas em $75 \mathrm{~mL}$ de água, em função de diferentes períodos de embebição.

O índice de velocidade de germinação mostrou resultados coerentes com o teste de condutividade elétrica em algumas combinações. O teste de condutividade elétrica tem se mostrado eficiente para avaliação do vigor de sementes de algumas espécies florestais. Conforme estudos realizados por Barbedo e Cícero (1998), para Inga uruguensis; Zucareli (1999) para Albizia hasslerii; Marques (2001) para Dalbergia nigra. No entanto, este teste também não 
foi considerado eficiente para Citharexylum montevidense (LEONHARDT, 2000).

$\mathrm{O}$ insucesso desse teste tem sido atribuído a vários fatores, dentre eles, as características do tegumento das sementes, podendo se constituir na principal causa de variações das informações obtidas quando são avaliadas sementes de diferentes cultivares ou até amostras de um mesmo cultivar (McCORMAC; KEFFE, 1990). Outro fator que tem influenciado a condutividade elétrica é o armazenamento das sementes em baixas temperaturas. Segundo Vieira et al. (2001) o armazenamento das sementes de soja a $10^{\circ} \mathrm{C}$, aparentemente estabilizou a integridade das membranas o que resultou em nenhuma mudança na condutividade elétrica das sementes. Pode-se também, levantar a hipótese de que o armazenamento das sementes em câmara fria, como também, a própria escarificação das sementes em ácido sulfúrico, pode ter influenciado nos resultados do teste de condutividade elétrica.

No trabalho, o teste de condutividade elétrica mostrou-se menos adequado para avaliação do potencial fisiológico dos lotes, embora a combinação de 75 sementes com $50 \mathrm{~mL}$ de água tenha apresentado resultados coerentes com os outros testes. Assim, recomendam-se outros estudos, envolvendo um maior número de lotes, visando fornecer subsídios que possam esclarecer a eficiência do teste na diferenciação do potencial fisiológico das sementes de mutambo.

Os resultados encontrados também confirmam a idéia descrita por outros pesquisadores e já comentada anteriormente, de que é interessante pesquisar tanto lotes com diferenças estreitas como outros com variação mais ampla no potencial fisiológico, porque há possibilidade de detectar a sensibilidade dos testes estudados, como também, fazer uso de vários testes para determinar o potencial fisiológico de lotes de sementes.

\section{Conclusões}

No teste de condutividade elétrica o uso de 75 sementes, com 50 ou $75 \mathrm{~mL}$, foi possível constatar que os períodos de embebição de seis horas e quatro horas mostraram concordância quanto à ordenação dos lotes. Entretanto, são necessários outros estudos, envolvendo um maior número de lotes, visando à possibilidade de uso como teste de vigor em sementes de mutamba.

Os testes de primeira contagem e índice de velocidade de germinação mostraram-se adequados para avaliação do potencial fisiológico dos lotes;

As sementes do lote III provenientes do município de Santa Rita do Passa Quatro, apresentou potencial fisiológico superior aos demais lotes estudados.

\section{Referências}

AMARAL, D. M. I..; VILLELA, F. A.; PESKE, S. T. Teste de condutividade elétrica e de lixiviação de potássio na avaliação da potencial fisiológico de sementes de louro (Cordia trichotoma (Vell.) Arrab. Ex Steud.) Boraginaceae. Informativo ABRATES, Curitiba, v. 7, n. 1/ 2, p. 215, 1997.

ANDERSON, J. D.; BAKER, J. E. Deterioration of seeds during aging. Phytopathology, Saint Paul, v. 73, n. 2, p. 321-325, 1983.

ARAÚJO NETO, J. C. Caracterização e germinação de sementes e desenvolvimento pós-seminal de mutamba (Guazuma ulmifolia Lam.). 1997. Dissertação (Mestrado em Produção e Tecnologia de Sementes) - Faculdade de Ciências Agrárias e Veterinárias, Universidade Estadual Paulista, Jaboticabal.

ASSOCIATION OF OFFICIAL SEED ANALYSTS. Seed vigor testing handbook. East Lasing: AOSA, 1983. (Contribution, 32).

BARBEDO, C. J.; CICERO, S. M. Utilização do teste de condutividade elétrica para previsão do potencial germinativo de sementes de ingá. Scientia Agricola, Piracicaba, v. 55, n. 2, p. 249-259, 1998.

BEWLEY, J. D.; BLACK, M. Seeds physiology of development and germination. New York: Plenum Press, 1994. 
BRASIL. Ministério da Agricultura. Regras para análise de sementes. Brasília: SNDA/DNDV/CLAV, 1992.

CARVALHO, N. M.; NAKAGAWA, J. Sementes: ciências, tecnologia e produção. 4.ed. Jaboticabal: FUNEP, 2000.

DIAS, D. C. F. S. Teste de condutividade elétrica e de lixiviação de potássio para avaliação do vigor de sementes de soja (Glycine max (L.) Merrill). 1994. Tese (Doutorado em Agronomia) - Escola Superior de Agricultura “Luiz de Queiroz", Universidade de São Paulo, Piracicaba.

DIAS, D. C. F. S.; VIEIRA, A. N.; BHÉRING, M. C. Estudo dos testes de condutividade elétrica e lixiviação de potássio para avaliação do vigor de sementes de hortaliças: 1. Couve-flor, cebola e cenoura. In: SEMINÁRIO PANAMERICANO DE SEMILLAS, 15., 1996, Gramado. Anais... Gramado: CESM FELAS, 1996. p. 28.

DUBOC, E.; SANTANA, D. G.; CARVALHO, M.; VIEIRA, M. G. G. C. Testes de vigor na avaliação da qualidade fisiológica de sementes de Leucaena leucocephala. Informativo ABRATES, Curitiba, v. 3, n. 3, p. 120, 1993.

HALMER, P.; BEWLEY, J. D. A physiological perspective on seed vigour testing. Seed Science and Techonology, Zurich, v. 12, n. 2, p. 561-575, 1984.

HAMPTON, J. G. Vigour testing within laboratories of the International Seed Testing Association: a survey. Seed Science and Techonology, Zurich, v. 20, Suppl. 1, p. 199203, 1992.

HAMPTON, J. G.; TEKRONY, D. M. Handbook of vigour test methods. 3.ed. Zurich: ISTA, 1995.

KRZYZANOWSKI, F. C.; FRANÇA NETO, J. B. Situação atual do uso de testes de vigor como rotina em programas de sementes no Brasil. Informativo ABRATES, Curitiba, v. 1, n. 3, p. 42-53, 1991.

LEONHARDT, C. Maturação fisiológica de sementes de tarumã-de-espinho (Citharexylum montevidense (Spreng.) Moldenke - Verbenaceae). 2000. Dissertação (Mestrado em Ciência e Tecnologia de Sementes) Faculdade de Agronomia "Eliseu Maciel”, Universidade Federal de Pelotas, Pelotas.

LOEFFLER, T. M. The bulk conductivity test as an indicator of soybean seed quality. 1981. Dissertation (M.S.) - University of Kentucky, Lexington.

LOEFFLER, T. M.; TEKRONY, D. M.; EGLI, D. B. The bulk conductivity test as an indicator of soybean seed quality. Journal of Seed Technology, Lansing, v. 12, n. 1, p. 37-53, 1988.

LOOMIS, E. L.; SMITH, O. The effect of artificial aging on the concentration of $\mathrm{Ca}, \mathrm{Mg}, \mathrm{Mn}, \mathrm{K}$, and $\mathrm{Cl}$ in imbibing cabbage seed. Journal American Society of Horticultural Science, Alexandria, v. 105, n. 5, p. 647-650, 1980.
MAGUIRE, J. D. Speed of germination-aid in selection and evaluation for seedling emergence and vigor. Crop Science, Madison, v. 2, n. 1, p. 176-177, 1962.

MARCOS FILHO, J. New approaches to seed vigor testing. Scientia Agricola. Piracicaba, v. 55, p.27-33, 1998. Número especial.

MARCOS FILHO, J. Testes de vigor: importância e utilização. In: KRZYZANOWSKI, F. C.; VIEIRA, R. D.; FRANÇA NETO, J. B. Vigor de sementes: conceitos e testes. Londrina: ABRATES, 1999.

MARCOS FILHO, J.; SILVA, W. R.; NOVEMBRE, A. D. C.; CHAMMA, H. M. C. P. Estudos comparativos de métodos para avaliação da qualidade fisiológica de sementes de soja, com ênfase ao teste de condutividade elétrica. Pesquisa Agropecuária Brasileira, Brasília, v. 25, n. 12, p. 1805-1815, 1990.

MARQUES, M. A. Teste de condutividade elétrica para avaliação da qualidade fisiológica de sementes de Dalbergia nigra Fr. Allem. (Jacarandá-da-bahia). 2001. Dissertação (Mestrado em Produção e Tecnologia de Sementes) - Faculdade de Ciências Agrárias e Veterinárias - Universidade Estadual Paulista, Jaboticabal.

McCORMAC, A. C.; KEFFE, P. D. Cauliflower seed vigour: imbibition effects. Journal of Experimental Botany, Oxford, v. 41, n. 228, p. 893-899, 1990.

MORAES, M. L. T.; OLIVEIRA, S. A.; FREITAS, M. L. M.; SÁ, M. E. Desempenho de sementes de duas populações de aroeira (Myracrodruon urundeuva Fr. All.) Anacardiaceae. Informativo ABRATES, Curitiba, v. 7, n. 1/ 2,p. 199, 1997.

MURPHY, J. B.; NOLAND, T. L. Temperature effects on seed imbibition and leakage mediated by viscosity and membranes. Plant Physiology, Bethesda, v. 69, n. 2, p. 428-431, 1982.

NAKAGAWA, J. Testes de vigor baseados no desempenho das plântulas. In: KRZYZANOWSKI, F. C.; VIEIRA, R. D.; FRANÇA NETO, J. B. (Ed.). Vigor de sementes: conceitos e testes. Londrina: ABRATES, 1999. cap. 2, p. 1-24.

NISIZAKI, S. M. A.; ZANGARO FILHO, W. Efeitos da inoculação de fungos micorrízicos arbusculares indígenas, no desenvolvimento de 12 espécies arbóreas nativas do Rio Tibagi, PR. In: CONGRESSO NACIONAL DE BOTÂNICA, 47., 1996, Nova Friburgo. Resumos... Nova Friburgo: Sociedade Botânica do Brasil, 1996. p. 385.

PAIVA AGUERO, J. A. Correlação de condutividade elétrica e outros testes de vigor com a emergência de plântulas de soja em campo. 1995. Dissertação (Mestrado em Produção e Tecnologia de Sementes)- Faculdade de Ciências Agrárias e Veterinárias, Universidade Estadual Paulista, Jaboticabal. 
PAULA, E. P.; ALVES, J. L. H. Madeiras nativas: anatomia, dendrologia, dendrometria, produção e uso. Brasília: Fundação Mokiti Okada, 1997.

PIANA, Z.; TILLMANN, M. A. A.; MINAMI, K. Avaliação da qualidade fisiológica de sementes de cebola e sua relação com a produção de mudas vigorosas. Revista Brasileira de Sementes, Brasília, v. 17, n. 2, p. 149-153, 1995.

PIÑA-RODRIGUES, F. C. M.; VALENTINI, S. R. T. Aplicação do teste de vigor em sementes. In: SILVA, A.; PINÃ-RODRIGUES, F. C. M.; FIGLIOLIA, M. B. (Coord.). Manual técnico de sementes florestais. São Paulo: Instituto Florestal, 1995. p. 74-84.

PRETE, C. E. C. Condutividade elétrica do exsudato de grãos de café (Coffea arabica L.) e sua relação com a qualidade da bebida. 1992. Tese (Doutorado em Fitotecnia) - Escola Superior de Agricultura "Luiz de Queiroz", Universidade de São Paulo, Piracicaba.

ROBERTS, E. H. Loss of viability: ultrastructural and physiological aspects. Seed Science and Technology, Zurich, v. 1, n. 2, p. 529-545, 1973.

TAO, J. K. Factors causing variations in the conductivity test for soybean seeds. Journal of Seed Technology, Lansing, v. 3, n. 1, p. 10-18, 1978.

TEKRONY,D. D.; EGLI, D. B. Relationship between laboratory indices of soybean seed vigor and field emergence. Crop Science, Madison, v. 17, n. 4, p. 573-577, 1977.
TORRES, S. B.; CASEIRO, R. F.; RODO, A. B.; MARCOS FILHO, J. Testes de vigor em sementes de maxixe (Cucumis anguria L.) com ênfase ao teste de condutividade elétrica. Revista Brasileira de Sementes, Brasília, v. 20, n. 2, p. 480483, 1998.

TRAPE, M. Z.; OLIVEIRA, C. Fichas de espécies nativas. Florestar Estatístico, São Paulo, v. 2, n. 6, p. 71-77, 1995.

VALENTINI, S. R. T.; PIÑA-RODRIGUES, F. C. Aplicação do teste de vigor em sementes. IF Série Registro, São Paulo, n. 14, p. 75-84, 1995.

VANZOLINI, S.; NAKAGAWA, J. Teste de condutividade elétrica em sementes de amendoim: efeitos de teor de água inicial e de períodos de embebição. Revista Brasileira de Sementes, Brasília, v. 21, n. 1, p. 46-52, 1999.

VIEIRA, R. D.; TEKRONY, D. M.; EGLI, D. B.; RUCKER, M. Electrical conductivity of soybean seeds after storage in several environments. Seed Science and Technology, Zurich, v. 29, n. 3, p. 599-608, 2001.

ZUCARELI, C. Desenvolvimento de testes rápidos para avaliação da qualidade fisiológica de sementes de Albizia hasslerii (Chodat) Burr. Leguminosae (Mimosaceae). 1999. Trabalho de Conclusão de Curso (Graduação de Agronomia) - Universidade Estadual do Oeste do Paraná, Marechal Cândido Rondon. 